\title{
Circle-Block Local Binary Patterns for Face Recognition
}

\author{
TANG Yifan, ZHAO Cairong, MIAO Duoqian \\ Department of Computer Science and Technology, Tongji University, China \\ tangyifanyifan@sina.com
}

Keywords: Circle-Block; Local Binary Patterns; Face Recognition.

Abstract. In this paper a new texture operator based on Local Binary Patterns (LBP) was proposed for face recognition called Circle-Block Local Binary Patterns (CB-LBP). The mean gray values of a circle block pixels are calculated to achieve macroscopic feature extraction. Our method was compared with the LBP-based patterns tested on Extended Yale B face image databases. Experimental results show the effectiveness of the proposed method.

\section{Introduction}

Local binary patterns (LBP), proposed by Ojala et al. in 2002 [1], has been successfully applied to facial expression analysis [2] and face recognition [3] owning to its computational simplicity and high efficiency [4]. By comparing the gray value of the central pixel to its neighborhood, LBP operator labels each result as 0 (less than) or 1 (greater than or equal to) [5]. Gray-scale invariant, rotation invariant and uniform LBP were introduced in succession by the same author [6]. Since then, many extensions and modifications have been made to improve the performance of the original LBP [7]-[11]. In multi-block LBP (MBLBP) [12][13], multi-scale mean filters are applied to capture robust macroscopic information.

The objective of this research is to improve the multi-block LBP operator using a novel circle-block local texture patterns computing the average pixel value of each center and each neighbor. The rest of the papers is organized as follows: In Section 2, we briefly review the original LBP multiresolution feature extraction. In Section 3 the details of the proposed Circle-Block LBP method are presented. We evaluate the effectiveness with extensive experiment on Extended Yale B face image database in Section 4. Finally, a conclusion is drawn in Section 5.

\section{Local binary patterns (LBP)}

A multiresolution approach is proposed by Ojala et al. [6] encoding a circularly symmetric set of pixels in a local neighborhood. It can be described by the new following formula:

$$
L B P_{P, R}=\sum_{p=1}^{P} s\left(g_{p}-g_{c}\right) 2^{p-1}, s(x)=\left\{\begin{array}{l}
1, x \geq 0 \\
0, x<0
\end{array}\right.
$$

where $P$ is the size of the neighbor set of pixels, $R$ is the radius of the local region, $g_{c}$ represents the gray value of the center pixel and $g_{p}(p=1,2, \ldots, P)$ denotes those of the neighbors. Suppose the coordinate of $g_{c}$ is $(0,0)$, then the coordinates of $g_{p}$ is $\left(R \cos \left(\frac{2 \pi p}{P}\right), R \sin \left(\frac{2 \pi p}{P}\right)\right)$. The grey values of neighbor pixels that are not in the center of image grids are approximately calculated by bilinear interpolation.

\section{Circle-Block Local Binary Patterns}

\section{Extract CB-LBP features}

Inspired by [12], we choose a circle round block area around each local center and every neighbor. The mean value of the circles is set as the comparator while the value of local center circle as an extra feature to be compared. Given the number of neighbors $P$ in a local patch, we treat each p-thneighbor pixel as a micro center $g_{p}$ and determine its $P$ sub-neighbor pixels $g_{p}^{i}$ on a circle of radius $R_{c}$. The 
sub-neighbor pixels of both the local center $g_{c}$ and its micro center $g_{p}$ in the neighborhood are taken into computation. The Circle-Block LBP (CB-LBP) operator is defined as follows:

$$
\begin{gathered}
C B-L B P_{P, R}=s\left(g_{c}^{m}-g_{M}\right) 2^{P}+\sum_{p=1}^{P} s\left(g_{p}^{m}-g_{M}\right) 2^{p-1}, \\
g_{c}^{m}=\frac{1}{P+1}\left(g_{c}+\sum_{i=1}^{P} g_{c}^{i}\right), g_{p}^{m}=\frac{1}{P+1}\left(g_{p}+\sum_{i=1}^{P} g_{p}^{i}\right) \\
s(x)=\left\{\begin{array}{l}
1, x \geq 0 \\
0, x<0
\end{array}\right.
\end{gathered}
$$

where $g_{p}^{i}$ and $g_{p}^{m}$ are the value of each pixel and the mean value in the p-th neighbor circle. $g_{c}^{i}$ and $g_{c}^{m}$ represent the value of each pixel and the mean value in the center circle and $g_{M}$ is the total mean value including all the calculated pixel values. Enlightened by [8] and [12], we take $g_{M}$ as the comparator to encode image robustly and $g_{c}^{m}$ as a feature to emphasize the importance of the local center.

\section{Radius $\boldsymbol{R}_{\mathrm{c}}$ and neighbors $n$}

The radius of the neighbor circle $R_{\mathrm{c}}$ should be adjusted according to the number of neighbors $n$ and radius $R$. It is easy to prove that when $R_{\mathrm{C}}$ satisfies the equation $R_{c}=R \sin \frac{180^{\circ}}{n}$, all of the neighbor circles are perfectly tangent to their two nearby circles.

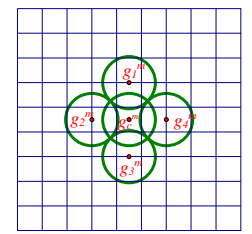

(a) $\mathrm{P}=4$

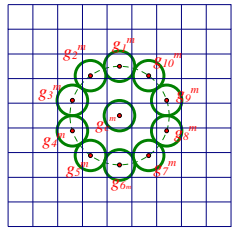

(b) $\mathrm{P}=10$

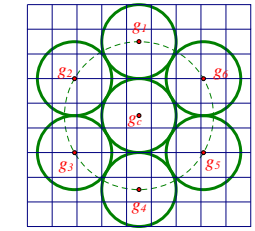

(c) $\mathrm{P}=6$

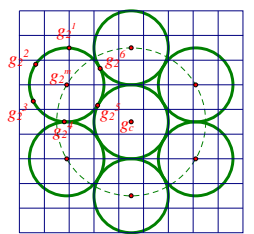

(d) $\mathrm{P}=6$

Figure 1.Different neighborhood size in Circle-Block LBP.

There are different ways to choose the number of neighbors $n$ as illustrated in Figure 1. In Figure 1(a) there are significant overlaps and the center resulting in unavoidable redundant information. In Figure 1(b) many blanks remain which fails to capture enough useful information. In Figure 1 (c) $R_{\mathrm{C}}$ is equal to $R / 2$. All the neighboring circles are tangent to the center circle if and only if $P=6$ where the feature dimension becomes $2^{6}=64$, as is demonstrated in Figure $1(\mathrm{~d})$. But 64 bins are not enough to form an descriptive histogram. So we choose 7-8 as the neighborhood size in our experiment.

\section{Comparison between encoded images}

L1 distance is adopted to measure the similarity of different face images. As is shown in Figure 2, two images of the same subject are depicted in the picture (a) (f), then encoded with LBP operator in (b)(g) and with CB-LBP with different radius in the picture (c)-(e) and (h)-(j). For LBP encoded images, the edges of light in (b) are more apparent than those in (g), while for CB-LBP encoded images the lighting variation between two subjects is much smaller. Details in eyes, nose and mouths are illustrated clearer in CB-LBP. It is easy to notice that as the radius R increases, the edges and angles of the senses become much more remarkable. 


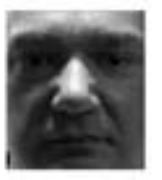

(a)

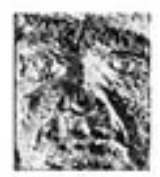

(b)

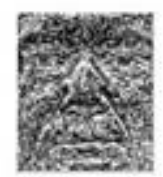

(c)

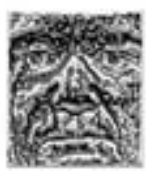

(d)

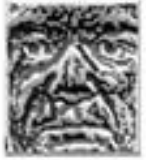

(e)

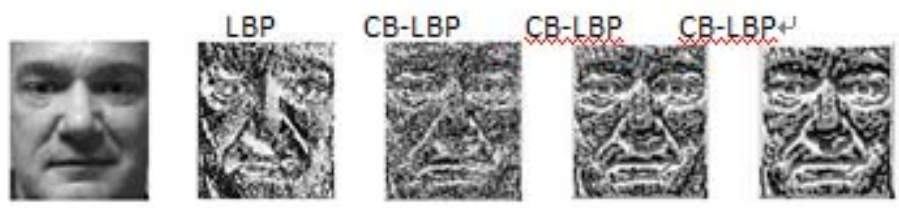

Figure 2. Comparison between two images and the encoding results using LBP and CB-LBP operators.

\section{Experiment}

\section{Parameter description}

In the following experiments, the optimal parameters of the compared algorithms are listed as follows. For all the LBP-based features, the neighbor size is 8 and the sub-block size is $7 \times 7$, while for CB-LBP the neighbor size keeps 7. Each block is with a 59-D histogram. The parameters of three patch based LBP (TPLBP) descriptors are $r=2, S=8$, and $w=3$. For the LTP features, the threshold for coding is 0.02. For LPQ, the local window size is 3 in all experiments.

\section{Experiment on Extended Yale B database}

There are 38 human subjects under 9 poses and 64 illumination conditions in the extended Yale B face image database. 60 frontal-face images of each subject are used in this experiment and each is resized to $96 \times 84$ and $48 \times 42$ pixels. We randomly choose $\mathrm{K}$ samples from each subject as a class for training and the remaining for test. Here, $K$ varies from 4 to 24 with an interval of 4 . For each $K$, we perform 10 runs of tests for each tested method and compute the average recognition rate as the result. Additionally, the standard NN classifier is employed for classification. Figure 3 shows the average recognition rate curve versus the variation of training sample size of $96 \times 84$ images after 10 runs.

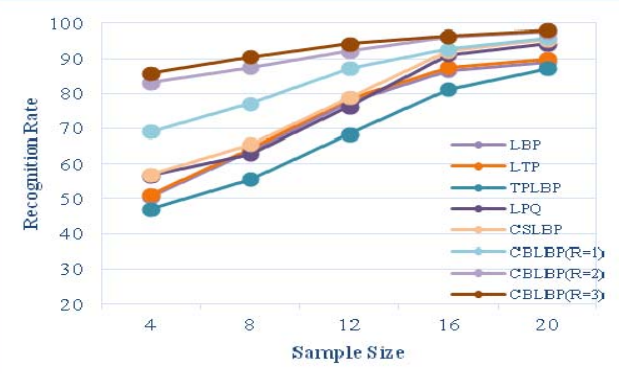

Figure 3. Average recognition rate curve versus the variation of training sample size using $96 \times 84$ images tested on Extended Yale B database.

From Figure 3, although TPLBP is not robust for face recognition especially under such difficult lighting conditions. LBP and LTP descriptors achieve better results for their general adaptability. CSLBP and LPQ descriptors slightly outperform the above two when the sample number $\mathrm{K}$ is large enough to sustain a stable extraction. However, these four descriptors are still not satisfying especially when the sample number $\mathrm{K}$ is small, since they all capture the local structural information by measuring point-to-point differences in a local region. CBLBP consistently achieves the best results especially because it covers a wide range of local pixels to gain a more robust recognition ability than the point-to-point methods above. $\mathrm{R}=3$ is the best radius configuration, for the recognition result achieves best and smoothly along with the change of sample size. 


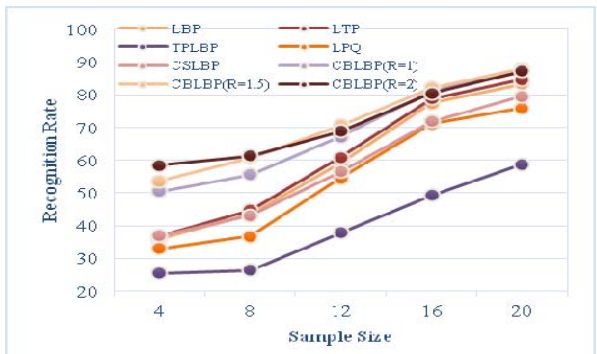

Figure 4. Maximum recognition rate curve versus the variation of training sample size using $48 \times 42$ images on Extended Yale B database.

Figure 4 gives a demonstration of recognition results on $48 \times 42$ images without modifying any other parameters. As the quality of images decreases, still CBLBP performs relatively well especially on a small sample size. The robustness of each algorithm meets a challenge thus the curves are not smooth. It should be noted that the curve of $\mathrm{R}=1.5$ runs ahead of other radius on the specific image size partly compared with $\mathrm{R}=3$ in the former experiment because of the compression of images. The performances of other descriptors are heavily influenced when the sample size is not large enough to extract adequate features.

\section{Conclusion}

In this paper, we develop a novel image feature extraction method called Circle-Block Local Binary Patterns for face recognition. The local structural information is exploited by measuring the local pixel difference between the average pixel values within a circle of a central pixel and those of its neighbor circles. CB-LBP exceeds the traditional LBP-based methods on its ability to adapt expression and illumination changes and its resistance to noise. Different block sizes and shapes will be combined together appropriately to achieve better results in the future studies.

\section{References}

[1] Ojala T, Pietikä M, Harwood I A D. A comparative study of texture measures with classification based on featured distribution. Pattern Recognition, 1996, (95):51-59.

[2] G, Zhao, Pietikäinen M. Dynamic Texture Recognition Using Local Binary Patterns with an Application to Facial Expressions. IEEE Transactions on Pattern Analysis \& Machine Intelligence, 2007, 29(6):915-928.

[3] Ahonen T, Hadid A, Pietikainen M. Face Description with Local Binary Patterns: Application to Face Recognition. IEEE Transactions on Pattern Analysis \& Machine Intelligence, 2006, 28(12):2037 - 2041.

[4] Maenpaa, T, Pietikainen M. Texture Analysis with Local Binary Patterns. Handbook of Pattern Recognition \& Computer Vision Edition (2005).

[5] Liao W, Young T. Texture Classification Using Uniform Extended Local Ternary Patterns. //International Symposium on Multimedia. IEEE, 2010:191-195.

[6] Ojala T, Pietikainen M, Maenpaa T. Multiresolution gray-scale and rotation invariant texture classification with local binary patterns. Pattern Analysis \& Machine Intelligence IEEE Transactions on, 2002, 24(7):971 - 987.

[7] Hafiane, A, and Guna S, Bertrand Z. science L N I C. Lecture notes in computer science. Advances in cryptology - EUROCRYPT 2014 : 33rd Annual International Conference on the Theory and Applications of Cryptographic Techniques Copenhagen, Denmark, May 11-15, 2014 : proceedings, 1984, 16(18):15--30. 
[8] Jin H L, Liu Q S, Lu H Q, Tong X F. Face detection using improved LBP under Bayesian framework. In: Proceedings of the 3rd International Conference on Image and Graphics. Hong Kong, China: IEEE, 2004. 306¡309

[9] Guo, Z.H., Zhang, L., Zhang, D. A Completed Modeling of Local Binary Pattern Operator for Texture Classification. Image Processing IEEE Transactions on, 2010, 19(6):1657 - 1663.

[10]Heikkilä M, Pietikäinen M, Schmid C. Description of interest regions with local binary patterns. Pattern Recognition, 2009, 42(3):425-436.

[11] Jin H, Liu Q, Lu H, et al. Face detection using improved LBP under Bayesian framework. //IEEE First Symposium on Multi-agent Security \& Survivability. IEEE, 2004:306 - 309.

[12]Liao S, Zhu X, Lei Z, et al. Learning Multi-scale Block Local Binary Patterns for Face Recognition/ Advances in Biometrics. Springer Berlin Heidelberg, 2007:828-837.

[13]Zhang L, Chu R, Xiang S, et al. Face Detection Based on Multi-Block LBP Representation/ Advances in Biometrics. Springer Berlin Heidelberg, 2007:11-18. 